\title{
CAUSAS DE CONTAGIO POR COVID 19 EN INTERNOS DE ENFERMERÍA
}

AUTORES: Tommy Israel Loor Quiñonez ${ }^{1}$

María del Rosario Herrera Velázquez ${ }^{2}$

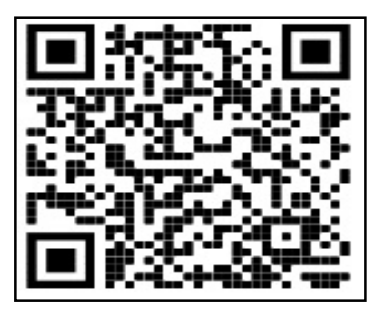

DIRECCIÓN PARA CORRESPONDENCIA: (tommyloor97@hotmail.com)

Fecha de recepción: 01/09/2020

Fecha de aceptación: 28/10/2020

\section{RESUMEN}

El coronavirus (COVID-19) es una enfermedad infecciosa causada por un coronavirus recientemente descubierto. Se analizan las causas por contagio de COVID-19 en los internos de enfermería en las instituciones asistenciales donde se encuentran brindando sus servicios. Para determinar, cuales son los errores que se cometen al momento de aplicar el protocolo de bioseguridad dispuesto por el ministerio de salud pública, para disminuir y prevenir contagios. Como causa probable de contagio se determinó el no uso de la mascarilla adecuada para el área y labor que estaban desempeñando, en 11 internos de enfermería correspondiente al 21\%. No colocar ni retirar bien las prendas de protección personal, tal como lo indica el protocolo de bioseguridad del MSP. Se definió como sintomático positivo a los internos que confirmaron su diagnóstico tras haberse realizado los exámenes complementarios de test inmunológico y pruebas rápidas. Las causas por las cuales los internos de enfermería se contagiaron por COVID-19 fue el uso incorrecto de las medidas de bioseguridad y prendas de protección personal en el área de salud donde se encontraban prestando sus servicios a la comunidad.

PALABRAS CLAVE: coronavirus; bioseguridad; protocolos; causas.

\section{CAUSES OF COVID-19 SPREAD IN NURSING INMATES}

\begin{abstract}
Coronavirus (COVID 19) is an infectious disease caused by a recently discovered coronavirus. The causes of contagion of covid19 in nursing interns in the healthcare institutions where they are found are analyzed to determine what errors are made when applying the biosafety protocol established by the Ministry of Public Health, to reduce and prevent contagion. As a probable cause of contagion, the non-use of the appropriate mask for the area and work they were

\footnotetext{
${ }^{1}$ Estudiante pre-profesional de la Carrera de Enfermería en la Universidad Estatal del Sur de Manabí. Provincia de Manabí, cantón Jipijapa- Ecuador. Correo: tommyloor97@hotmail.com

${ }^{2}$ Docente Titular 2 de la Carrera: Enfermería, Facultad de Ciencias de la Salud de la Universidad Estatal del sur de ManabíE-mail: mariarosario.herrera@unesum.edu
} 
Tommy Israel Loor Quiñonez, María del Rosario Herrera Velázquez

performing was determined, in 11 nursing inmates corresponding to $21 \%$, not putting on or removing the personal protective garments properly, as indicated in the protocol biosafety department. Inmates who confirmed their diagnosis after having undergone complementary immunological tests and rapid tests were defined as positive symptomatic. The causes for which the nursing interns were infected by covid19 was the incorrect use of biosecurity measures and personal protective clothing in the health area where they were providing their services to the community

KEYWORDS: coronavirus biosecurity; protocols; causes.

\section{INTRODUCCIÓN}

La COVID19 es una enfermedad infecciosa causada por el coronavirus SARS CoV que se ha descubierto recientemente. Tanto este nuevo virus como la enfermedad que provoca eran desconocidos antes de que estallara el brote en Wuhan (China) en diciembre de 2019. Actualmente la COVID-19 es una pandemia que afecta a muchos países de todo el mundo (OMS 2019).

Los síntomas más habituales de la COVID-19 son la fiebre, la tos seca y el cansancio. Otros síntomas menos frecuentes que afectan a algunos pacientes son los dolores y molestias, la congestión nasal, el dolor de cabeza, la conjuntivitis, el dolor de garganta, la diarrea, la pérdida del gusto o el olfato y las erupciones cutáneas o cambios de color en los dedos de las manos o los pies. Estos síntomas suelen ser leves y comienzan gradualmente. Algunas de las personas infectadas solo presentan síntomas leves (OMS 2019).

Si bien es claro, se está buscando una vacuna que logre detener la propagación de la pandemia todavía no se ha logrado producir y comercializar este antídoto por lo que no tenemos tratamiento establecido para ser utilizado. Situación de COVID-19 en la Región de las Américas, 27 de agosto de 2020. Se notificaron 135.430 casos adicionales y 4.004 muertes en las últimas 24 horas, lo que representa un aumento relativo de $1.06 \%$ en casos y un aumento relativo de muertes de $0.89 \%$, en comparación con el día anterior. Tras el brote de una enfermedad por un nuevo coronavirus (COVID-19) que se produjo en Wuhan, una ciudad de la provincia de Hubei, en China, se ha registrado una rápida propagación a escala comunitaria, regional e internacional, con un aumento exponencial del número de casos y muertes.

El 30 de enero del 2020, el Director General de la OMS declaró que el brote de COVID-19 era una emergencia de salud pública de importancia internacional de conformidad con el Reglamento Sanitario Internacional (2005). El primer caso en la Región de las Américas se confirmó en Estados Unidos el 20 de enero del 2020, y Brasil notificó el primer caso en América Latina y el Caribe el 26 de febrero del 2020. Desde entonces, la COVID-19 se ha propagado a los 54 países y territorios de la Región de las Américas (OMS, 2020).

En Ecuador, desde el caso 0 del 29 de febrero del 2020 la curva de contagio no ha disminuido pese a las medidas que establece el Ministerio de Salud Pública, para la ciudadanía en general y protocolos de contingencia y bioseguridad para los centros hospitalarios y personal de salud. Llegando a alcanzar, la cifra más alta de fallecidos el 10 de mayo del 2020 con 410 perdidas en un solo día según la información que proporciona el MSP diariamente (MSP, 2020).

60 UNESUM-Ciencias. Publicación cuatrimestral. Vol. 4, No. 3 (Número Especial), Año 2020. 
Según el comunicado que emite el gobierno de la situación nacional por la pandemia, actualmente se contabilizan 90,535 confirmados de los cuales 59.344 de aquellos pacientes están recuperados, 1.974 pacientes tienen el alta hospitalaria y 124.384 casos fueron descartados hasta el 6 de Agosto del 2020 se han registrado 5.877 personas fallecidas adicionalmente se registran 3.530 fallecidos, probables por Covid19 a nivel nacional. Hasta la fecha se han tomado 258.562 muestras de Par + pruebas rápidas, 384 están hospitalizados con pronóstico reservado y 833 están estables, monitorizados y tendidos en los hospitales (PUBLICA, 2020), (Contreras \& Sierra, 2020).

Por todo lo anterior, se analizan las causas por contagio de covid19 en los internos de enfermería en las instituciones asistenciales donde se encuentran para determinar, cuales son los errores que se cometen al momento de aplicar el protocolo de bioseguridad dispuesto por el ministerio de salud pública, para disminuir y prevenir contagios.

\section{DESARROLLO}

Se realizó un estudio analítico observacional de corte transversal en el mes de agosto del presente año, previo consentimiento verbal de los participantes. Para este trabajo investigativo se consideraron internos rotativos de la carrera de enfermería, Facultad Ciencias de la Salud de la Universidad Estatal del Sur de Manabí del cantón Jipijapa- Ecuador. El cuerpo de internos fue distribuido en distintas provincias tanto en áreas hospitalarias y comunitarias, centros de salud tipo A, B y C. Se tomaron como referencia para la aplicación de la encuesta, internos de enfermería del octavo y noveno semestre para la investigación en el período que abarca mayoseptiembre en el año 2020.

\section{Población}

La población total de los internos de enfermería de esa cohorte fueron 79 a los cuales se les informó el objetivo de estudio y aquellos que estaban de acuerdo llenaron la encuesta voluntariamente, con una muestra de 41 internos, a quienes se les envió el formulario de la herramienta de Google vía online a sus dispositivos electrónicos. El encuestador estuvo atento a cualquier interrogante durante los 10 minutos que tomo llenar la encuesta. Fueron excluidos los participantes que no deseaban llenar la encuesta o dejaban incompletas algunas preguntas.

Instrumento

La encuesta fue anónima y auto-administrada, con 20 interrogantes que incluyeron datos relacionados con causas asociadas a contagios por covid19, protocolos de bioseguridad del ministerio de salud pública y la organización mundial de la salud (OMS). Para evaluar las causas de contagios por covid19 y medidas de bioseguridad se utilizaron las escalas de opción múltiple, individual y lineal aprobada por la tutora de la asignatura redacción científica. Se analizaron las encuestas de los internos que habían pasado por la enfermedad y los que la tenían al momento de realizar la encuesta.

Definición de variables 
Tommy Israel Loor Quiñonez, María del Rosario Herrera Velázquez

Se definió como sintomático positivo a los internos que confirmaron su diagnóstico tras haberse realizado los exámenes complementarios de test inmunológico y pruebas rápidas. Para determinar las causas de contagio por covid-19, se utilizaron siete alternativas de opción múltiple, siendo estas el manejo de las normas de bioseguridad, la aplicación correcta de los cinco momentos del lavado de manos, la correcta colocación y retiro de las prendas de protección personal, uso completo de las prendas de protección personal, uso de mascarilla adecuada según el área de trabajo, manipulación del rostro mientras trabaja y finalmente se indagó sobre la forma en la que el interno infectado pudo haber contraído el virus causante de la enfermedad COVID-19.

Aspectos éticos

Se cuidó meticulosamente la privacidad y confiabilidad de todos los participantes del estudio, no se requirieron datos personales: nombres apellidos y números de cedulas. Los datos entregados anónimamente en los formularios no podrán ser accesibles ni usados por entes extraños a la investigación.

\section{RESULTADOS Y DISCUSIÓN}

De un total de 79 Internos de Enfermería se encuestó a 25 de ellos (la tasa de aceptación fué del $51.8 \%$ ). Como causa probable de contagio se determinó el no uso de la mascarilla adecuada para el área y labor que estaban desempeñando, en 11 internos de enfermería correspondiente al 21\%, no colocar ni retirar bien las prendas de protección personal, tal como lo indica el protocolo de bioseguridad del MSP. El 15\% no utilizaba completas las prendas de protección personal. Al no usar una mascarilla adecuada para manejar pacientes con esta enfermedad, el riego de contagio es muy alto.

Ya que al hablar, toser o estornudar se expulsa micro partículas que quedan suspendidas en el aire, estas no llegan muy lejos y se precipitan rápidamente al suelo. En las áreas donde se mantienen a los dependientes respiratorios el riesgo es mucho más alto, por esto se usan respiradores al realizar procedimientos a los pacientes evitando contagios (OMS, 2020), (VELAZQUEZ \& BENITEZ, 2020). Esto refuerza explícitamente lo que indica el protocolo de bioseguridad sobre el uso obligatorio de mascarillas y el cómo ponerse y sacar correctamente las prendas de protección personal, medidas dispuestas como prevención a futuros contagios.

Infectados por SARS-CoV2

El 78\% equivalente a 32 internos de enfermería no se encuentran infectados o ya pasaron la enfermedad sin complicación alguna. El 56\% igual a 23 internos indicó que sí se contagiaron por el virus, al menos una vez, desde que inició la pandemia, El 22\% de los encuestados estaba cursando por la enfermedad esto corroborado por un diagnóstico positivo para COVID-19 la medida a cumplir en estos casos es 15 días de aislamiento domiciliario obligatorio, el 44\% nunca ha contraído el virus por ningún motivo. La encuesta muestra que el $44 \%$ equivalente a 18 internos de enfermería si habían adquirido el virus en las áreas de la institución donde estuvieron prestando sus servicios.

Otro factor evaluado indicó que los internos si se contagiaron por el virus al menos una vez desde que iniciaron la pandemia. Desde el primer brote de la pandemia en nuestro país hasta que

62 UNESUM-Ciencias. Publicación cuatrimestral. Vol. 4, No. 3 (Número Especial), Año 2020. 
entraron a laborar como internos de enfermerías a las instituciones asistenciales de salud, sí llegaron a infectarse con el virus. Otros estudios afirman que es posible contagiarse por covid19 con otra persona que no presente los síntomas característicos de la enfermedad, aun no se sabe con qué frecuencia ocurre, pero la organización mundial de la salud este asiendo estudios sobre este tema en cuestión e indico que seguirá informando sobre las conclusiones que allá obtenido. La mayor parte de la población carece de autodisciplina, no acata disposiciones, el aislamiento es una medida de prevención al igual que el uso de la mascarilla y mantener distanciamiento social, ayudaría en gran parte a mitigar contagios solo con cumplir y hacer cumplir estas disposiciones (OMS, 2020), (Vega \& Marrufo, 2020).

\section{Diagnóstico}

Para el diagnóstico se utilizaron pruebas y exámenes complementarios, realizados por las instituciones de salud donde los internos se encontraban. Entre estos exámenes, el 39\% (17 internos) se realizó el test inmunológico de confirmación, la prueba rápida se la realizaron 15 internos (34\%) y finalmente 12 estudiantes (27\%) de internado no usó ningún método para confirmar el diagnóstico.

El diagnóstico microbiológico de las infecciones en fase aguda implica la detección del microorganismo por métodos directos como el cultivo y los métodos moleculares o utilizando métodos indirectos como la serología. En el caso de COVID-19, su diagnóstico microbiológico a nivel mundial se basa, en la detección del material genético (ARN) viral del SARS-CoV-2 mediante técnicas de PCR, en muestras respiratorias de pacientes con síntomas compatibles. El cultivo celular por su parte, no es un método de empleo rutinario para el diagnóstico. Gracias a este tipo de pruebas se puede diagnosticar la presencia del virus en el sistema inmunitario, descartando o afirmando el diagnóstico del mismo (PUBLICA, 2020), (Urzúa et al., 2020).(Cornelio \& Gulín, 2018).

Síntomas presentes durante la infección

Una de la sintomatología que más presentaron los internos de enfermería fue cefalea en el 27\% de los casos, el $20 \%$ de los internos encuestados manifestaron alza térmica, solo el $16 \%$ de los internos manifestaron sentir cansancio, el $10 \%$ de los internos sintomáticos presentaron tos y el $4 \%$ de los internos refirieron la disnea en su sintomatología. El 20\% de los encuestados refirió no presentar ningún síntoma ni malestar durante la infección, a pesar de resultar positivos en el examen de laboratorio.

Los síntomas más comunes de la COVID-19 son fiebre, cansancio y tos seca. Algunos pacientes pueden presentar dolores, congestión nasal, rinorrea, dolor de garganta o diarrea. La mayoría de las personas (alrededor del 80\%) se recupera de la enfermedad sin necesidad de realizar ningún tratamiento especial. Las personas mayores y las que padecen afecciones médicas subyacentes, como hipertensión arterial, problemas cardiacos o diabetes, tienen más probabilidades de desarrollar una enfermedad grave. En torno al 2\% de las personas que han contraído la enfermedad han muerto. La valoración temprana de los síntomas del paciente, es crucial para un diagnostico efectivo y seguro, y la aplicación de un tratamiento oportuno para mitigar los 
Tommy Israel Loor Quiñonez, María del Rosario Herrera Velázquez

síntomas. Ya que tratamiento definitivo para la enfermedad aún no hay (YIRGOGEN, 2020), (Cornelio et al., 2016).

\section{Protocolo de bioseguridad}

La investigación dio como resultado que 25 internos (61\%) conocen y aplican los protocolos de bioseguridad establecidos, sin embargo, resulta preocupante y hasta cierto punto alarmante, que un $37 \%$ de los encuestados, saben poco de esta importante medida de seguridad para evitar el contagio y la propagación de la enfermedad.

Los protocolos dispuestos por la Organización Mundial de la Salud, he implementados por el Ministerio de Salud Pública a nivel nacional, cumplen un papel importante al momento de interactuar con el paciente, siendo beneficioso para la salud del personal médico, y el resto de pacientes de la institución estas medidas son adoptadas por todas las instituciones de salud, el conocimiento de las mismas es imprescindible.

Bioseguridad, conjunto de medidas científico-organizativas, destinadas a proteger al trabajador, a la comunidad y al medio ambiente, de riesgos de trabajar con agentes biológicos o la liberación de organismos al medio ambiente, para disminuir los efectos que presenten y eliminar su consecuencia. La disposición de estos protocolos ha sido de gran importancia para el manejo de la pandemia, ayudando al personal de salud a auto-protegerse y hacer su trabajo más eficiente frente a pacientes que estén contagiados y necesiten de cuidados.(Arteta, 2012)

Uso correcto de los Equipos de Bioseguridad

Los equipos de bioseguridad son indispensables para prevenir y evitar contaminación en las áreas de atención, el 83\% correspondiente a 34 de los internos de enfermería manifestó que si utiliza correctamente los equipos de protección personal. El 17\% equivalente 7 internos no utiliza correctamente los equipos de bioseguridad.

Al comparar los resultados del nivel de conocimiento sobre medidas de bioseguridad en el personal del HNHU con estudios similares, encontramos que este tiende a ser inferior o semejante al de otros hospitales. Uno de los estudios presentados coincide con el hallazgo de un mayor nivel de conocimiento por parte del personal médico sobre el de enfermería. Este mismo coincide con un nivel de conocimiento semejante en ambos sexos. Resultados del estudio antes mencionado, y otro agregado, coincidieron en un menor nivel de conocimiento sobre bioseguridad relacionado a mayor edad y mayor tiempo laboral en una institución. Otro estudio presenta un porcentaje similar de personal capacitado sobre bioseguridad, mostrando además un nivel de conocimiento semejante.

Dos investigaciones presentaron personal con un nivel de conocimiento de bioseguridad bueno, tras haber sido capacitados. Esto no fue encontrado en nuestro estudio. Estas diferencias y semejanzas con otros estudios similares pueden estar expresando la necesidad de una capacitación sobre las medidas de bioseguridad más eficiente, además de dedicarle especial atención al personal de mayor edad y tiempo laborando en la institución (Bertocchi, 2017).

Equipos de protección personal utilizada a diario

64 UNESUM-Ciencias. Publicación cuatrimestral. Vol. 4, No. 3 (Número Especial), Año 2020. 
Los internos de enfermería utilizaron como prendas de protección personal el (18\%) descartable, el 16\% de los internos usaron gorro quirúrgico descartable como prenda de protección personal para la cabeza, el $12 \%$ de los encuestados utilizaban batas descartables, el $7 \%$ usaba mascarilla kn95 indicada para el uso del personal médico, el 6\% de los internos utilizaban zapatones descartables. El 21\% utilizaban el lavado de manos como medida de bioseguridad.

Los equipos de protección personal (EPP) en la actualidad se constituyen como uno de los conceptos más básicos en cuanto a la seguridad en el lugar de trabajo y son necesarios cuando los peligros no han podido ser eliminados por completo o controlados por otros medios. El uso diario de estos equipos es impórtate en la actividad diaria del profesional de alud que rinda cuidados directos a pacientes con sintomatología presente de covid19 (Andrés, 2016).

Uso de las Prendas de Protección Personal

El protocolo de bioseguridad dispuesto por el ministerio de salud pública a nivel nacional establece que el uso de la mascarilla quirúrgica descartable es de 4 horas su vida útil, el $50 \%$ de los internos entrevistados indicaron que utilizan la mascarilla 8 horas al día. El 30\% la utiliza 12 horas al día, solo el 15\% de todos los internos entrevistados las utilizan las 4 horas establecidas.

El $41 \%$ de los internos encuestados no se retiran nunca la mascarilla para darse un descanso y respirar, el 37\% de los encuestados se la retiran cada 2 horas para darse un descanso, el 12\% se la retira cada 60 minutos y el $10 \%$ de los internos se las retiran en un intervalo de 30 minutos y 6 horas.

La frecuencia en la cual se manipulan el rostro o sus prendas de protección facial después de colocárselas es del 70\% correspondiente a 28 de los internos y lo hacen 1 o 2 veces en el turno. El $20 \%$ de ellos nunca se toca el rostro y el $10 \%$ de los internos lo hace más de 5 veces en el turno.

El 41\% equivalente a 17 internos de enfermería siempre vuelven a reutilizar algún equipo de bioseguridad descartable por la falta de implementos y material de protección en las instituciones de salud pública donde están ubicados el $27 \%$ lo vuelve a reutilizar a veces medida obligatoria por la falta de recursos y el 32\% referente a 13 de 41 internos encuestados nunca vuelven a reutilizar un equipo de protección personal descartable que ya hayan utilizado. Además los que reutilizan manifiestan que antes de volverse a poner estas prendas la pasan por el esterilizador para matar bacterias, virus y microorganismos que puedan tener las mismas.

El protocolo de bioseguridad pone en manifiesto que cada procedimiento o atención de pacientes individualizados se deberá utilizar un par de guantes nuevos, el 80\% de los internos entrevistados utiliza un par de guantes por paciente El 10\% manifiesta que casi siempre utiliza un par de guantes, $10 \%$ solo lo hace si esta necesario o si realizara algún procedimiento que comprometa la manipulación de fluidos corporales.

El estudio demuestra que la mayoría de los centros disponen y tienen establecido cuando deben utilizarse los EPI específicos tales como: guantes, protecciones respiratorias, protecciones oculares, ropa de protección o de trabajo así como equipos para situaciones de emergencia. En más de un $90 \%$ de los centros se imparte formación y se informa a los trabajadores tanto cuanto se introducen cambios en la utilización de los EPI como también sobre las ventajas e 
inconvenientes de la vacunación. Sin embargo en cuanto a la documentación, el porcentaje baja hasta el $40 \%$ la existencia de algún documento que acredite la entrega de los EPI a los trabajadores y a un 78\% sobre procedimientos de información específicos sobre manipulación de sustancias infecciosas.

Los resultados obtenidos en este estudio, así como la reciente aparición de normativa técnica específica sobre EPI, ropa de protección y calzado de protección contra microorganismos inciden en la necesidad de seguir trabajando sobre este tema. Finalmente cabe resaltar la importancia de la labor de formación e información, ya que el personal expuesto a riesgos biológicos debe diferenciar claramente los EPI, de la ropa de trabajo y de los equipos destinados a la protección del producto (paciente o muestra manipulada), usándose éstos últimos como protecciones personales frente al riesgo biológico, cuando en la mayoría de situaciones no sólo no son eficaces sino que producen una situación de falsa seguridad (Angelina Constans Aubert, 2011).

Lavado de manos

El ministerio de salud pública prioriza en gran medida el lavado de manos obligatorio para todos los profesionales de salud en todas las instituciones a nivel nacional, entre estas medidas se colocan carteles donde específica y muestra el modo correcto que se debe hacer este procedimiento, para brindar atención tenemos los cinco pasos correctos. En este aspecto, todos los encuestados coinciden en la realización adecuada del proceso, el 100\% de los encuestados respondió, después de riesgo y exposición a líquidos y fluidos corporales, después de manipular al paciente, antes de manipular al paciente, antes de realizar cualquier procedimiento y después del contacto con el entorno del paciente. A parte del agua y jabón que un eje principal para este procedimiento se investigó si los internos de enfermería utilizaban alcohol para desinfectarse las manos, el 85\% correspondiente a 35 de los internos siempre se desinfecta con alcohol, el 15\% lo hace a veces.

El lavado de las manos se ha considerado con razón una medida de higiene personal durante siglos. Actualmente se dispone de suficiente evidencia científica que demuestra que esta medida sencilla y barata puede ayudar a reducir las infecciones de forma clínica y sanitariamente significativa. A pesar de ello, los estudios publicados muestran que la higiene de las manos se realiza sólo entre un 15 y un 50\% de las veces en las que debería llevarse a cabo. Con objeto de apoyar a los diferentes países en el establecimiento de prioridades para hacer frente a las infecciones relacionadas con la atención sanitaria, la Organización Mundial de la Salud ha desarrollado una campaña destinada a mejorar el cumplimiento la higiene de las manos. Para ello son elementos clave la formación del personal, el cambio de sus hábitos, su motivación y la accesibilidad a los productos en los puntos de atención a los pacientes. A nivel institucional es necesario que los gestores sanitarios se posicionen de forma manifiesta, situando la higiene de las manos como uno de los objetivos de calidad de la institución (T. Pi-Sunyer Cañellasa, 2015).

Desinfección de equipos médicos

El 90\% equivalente a 38 de los internos entrevistados respondió que desinfectan los equipos médicos cada vez que lo utiliza con un paciente, el $10 \%$ lo desinfecta solo 2 veces por turno, antes o después de cada turno. También señalaron que el $83 \%$ de los internos encuestados

66 UNESUM-Ciencias. Publicación cuatrimestral. Vol. 4, No. 3 (Número Especial), Año 2020. 
mantienen su lugar de trabajo desinfectado, ordenado agradable y en óptimas condiciones el 17\% respondió que limpian y desinfectan lo necesario.

El 95\% equivalente a 39 internos de enfermería maneja con estricta precaución objetos corto punsantes y el 5\% correspondiente a 2 de ellos lo hacen a veces, siendo de mucha importancia para nuestra seguridad el manejo correcto y adecuado de estos desechos previniendo enfermarnos de cualquier patología o dañándonos nosotros mismos.

En el ambiente los microorganismos están presentes en gran número en la humedad y sobre fómites, pero algunos de ellos pueden persistir bajo condiciones secas. La presencia del patógeno no establece su papel causal; su transmisión desde la fuente de huéspedes se puede hacer a través de medios indirectos por ejemplo a través de la transmisión de manos. Las superficies por lo tanto, deberían considerarse como uno de los reservorios potenciales más importantes que albergan patógenos, y la presencia de un huésped susceptible es uno de los componentes que subraya la importancia del ambiente en las infecciones asociadas al cuidado de la salud y los patógenos oportunistas en fómites, aire y agua; como resultado de los avances hechos en la tecnología y tratamientos médicos se aumenta el riesgo y los pacientes se vuelven susceptibles en el curso del tratamiento y por lo tanto enfrentan un riesgo aumentado de adquirir infecciones oportunistas en las instituciones de salud (Calderón, 2015).

\section{CONCLUSIONES}

Las causas por las cuales los internos de enfermería se contagiaron por COVID-19 fue el uso incorrecto de las medidas de bioseguridad y prendas de protección personal en el área de salud donde se encontraban prestando sus servicios a la comunidad, y presentaron la sintomatología correspondiente de dicha enfermedad corroborado su diagnóstico con una prueba de laboratorio.

\section{REFERENCIAS BIBLIOGRÁFICAS}

Andrés, E. M. (2016). NORMS Y PROTOCOLOS DE VIOSEGURIDAD. SCELO, 15.

Angelina Constans Aubert, R. M. (2011). Utilización de los equipos de protección individual frente al riesgo biológico por el personal sanitario. SCIELO, 19.

Arteta, I. G. (2012). Diseño e implementación del protocolo de bioseguridad. dialnet. 1-2.

Bertocchi, J. A. (2017). Conocimiento de las medidas de bioseguridad en personal de salud. scielo PERU. 3-12.

Calderón, M. E. (2015). SECRETARÍA DISTRITAL DE SALUD. BOGOTA POSITIVA, 12.

MSP. (2020). Situación coronavirus Covid-19 (infografía) . MINISTERIO DE SALUD PUBLICA ECUADOR, 1.2.

OMS. (2020, 31 JULIO). ORGANIZACION MUNDIAL DE LA SALUD. Recuperado el AGOSTO 15, 2020, 2-4. de https://www.who.int/es/emergencies/diseases/novel-coronavirus-2019

OMS, O. (2020). Informes de situación de la COVID-19. ORGANIZACION PANAMERICANA, ORGANIZACION MUNDIAL DE LA SALUD.1, 5-6.

PUBLICA, M. D. (2020). Actualización de casos de coronavirus en Ecuador. INFOGRAFIA .0-1. 
Tommy Israel Loor Quiñonez, María del Rosario Herrera Velázquez

\section{PUBLICA., M. D. (2020). PROTOCOLO DE USO DE PRUEBAS RÁPIDAS PARA DETECCIÓN DE} ANTICUERPOS. MSP, 1.

T. Pi-Sunyer Cañellasa, M. B. (2015). Higiene de las manos: evidencia científica y sentido común. ELSEVIER, 5659.

YIRGOGEN, H. (2020). (COVID 19) MEDIDAS DE PROTECCION SOBRE EL CORONAVIRUS. VISION, 9.

Contreras, B. N. M., \& Sierra, J. J. P. (2020). Reflexiones del cuidado enfermero en tiempos de COVID-19. Enfermería Investiga, 5(3), 71-78. https://revistas.uta.edu.ec/erevista/index.php/enfi/article/download/914/859

Cornelio, O. M., Beatriz, M., \& Rubido, C. (2016). Práctica de Microbiología y Parasitología Médica integrado al Sistema de Laboratorios a Distancia en la carrera de Medicina. Revista de Ciencias Médicas de Pinar del Río, 20(2), 174-181.

Cornelio, O. M., \& Gulín, J. G. (2018). Modelo para la evaluación de habilidades profesionales en un Sistema de Laboratorios a Distancia. Revista Científica, 3(33), 1.

Urzúa, A., Vera-Villarroel, P., Caqueo-Urízar, A., \& Polanco-Carrasco, R. (2020). La Psicología en la prevención y manejo del COVID-19. Aportes desde la evidencia inicial. Terapia psicológica, 38(1), 103-118. https://scielo.conicyt.cl/scielo.php?pid=S0718-48082020000100103\&script=sci_arttext

Vega, M. J. L., \& Marrufo, H. R. M. (2020). Situación de Enfermería en tiempos de COVID-19: Una mirada panorámica. Journal of America health, 3(3), 48-58. http://www.jahjournal.com/index.php/jah/article/download/50/106

VELAZQUEZ, C. A., \& BENITEZ, O. D. R. (2020). ACTITUDES DEL PERSONAL DE ENFERMERÍA EN EL HOGAR ANTE LA PANDEMIA COVID-19. AcademicDisclosure, 1(1), 262-272.

https://revistascientificas.una.py/ojs/index.php/rfenob/article/download/154/128

68 UNESUM-Ciencias. Publicación cuatrimestral. Vol. 4, No. 3 (Número Especial), Año 2020. 